\title{
Espécies silvestres alojadas no Centro de Triagem de Animais Silvestres/Acre: implicações conservacionistas
}

\section{Wild species housed in Animal Sorting Centers/Acre: conservationist implications}

\author{
Jucilene Silva do Nascimento ${ }^{1}$, Amanda Moura Badarane ${ }^{2}$, Michelline Medeiros de \\ Oliveira Dantas ${ }^{3}$, Adenhauer Silva Urbanski $^{4}$, Elaine Christina Oliveira do Carmo ${ }^{5}$, \\ Vânia Maria França Ribeiro ${ }^{6}$
}

\begin{abstract}
Resumo
Os Centros de Triagem de Animais Silvestres - CETAS são instituições que têm como função receber animais silvestres oriundos de cativeiros domésticos, apreensões dos órgãos fiscalizadores em operações de combate ao tráfico ou vítimas de acidentes ambientais, sendo $90,9 \%$ das unidades de responsabilidade do IBAMA e $9,1 \%$ de empresas privadas. O trabalho teve como objetivo realizar um levantamento das espécies de animais silvestres recebidos no CETAS de Rio Branco - Acre nos anos de 2010 a 2014, avaliando suas implicações conservacionistas. Durante esse período foram apreendidos 1.097 aves $(47,2 \%), 720$ répteis $(31,0 \%), 498$ mamíferos $(21,4 \%)$ e cinco peixes $(0,2 \%)$, totalizando 2.320 animais recepcionados, dentre eles, $0,6 \%$ estão presentes na lista de animais vulneráveis e ameaçados de extinção. A despeito das fiscalizações realizadas pelo IBAMA e policiamento ambiental, animais com risco de extinção continuam sendo retirados da natureza de forma predatória, sendo as aves as mais apreciadas que os demais grupos, considerando o número de animais recebidos pelo CETAS.
\end{abstract}

Palavras-chave: Órgãos ambientais. Resgate. Tráfico.

\begin{abstract}
The Wild Animal Sorting Centers - CETAS are institutions responsible for receiving wild animals. They animals were rescued from domestic captivity, from seizures during inspections by government agents in actions against illegal trafficking, and from environment accidents. The majority $(90.9 \%)$ are controlled by IBAMA and only $9.1 \%$ by the private sector. This worked aims at doing a survey of the species of wild animals received by the CETAS in Rio Branco - Acre from 2010 to 2014, and evaluating the conservationist implications involved. During that time span 2,320 animals were received, comprising 1,097 birds (47.2\%), 720 reptiles (31.0\%), 498 mammals (21.4\%) and 5 fish (0.2\%). From the animals rescued, $0.6 \%$ belong to the list of species threatened with extinction. Despite the inspections carried out by IBAMA and by the environment police, threatened species continue to be predatorily taken out from their habitats, especially birds, which are the most attacked group, considering the number of animals received for CETAS.
\end{abstract}

Keywords: Environment agencies. Rescue. Traffic.

${ }^{1}$ Engenheira Agrônoma. Mestranda em Sanidade e Produção Animal Sustentável na Amazônia Ocidental (MESPA), instituição (UFAC). E-mail: juci.jnascimento@gmail.com.

${ }^{2}$ Bióloga, especialista, docente do Instituto Federal de Educação, Ciência e Tecnologia do Acre (IFAC). E-mail: amanda.badarane@, ifac.edu.br.

${ }^{3}$ Médica Veterinária. Mestrando em Sanidade e Produção Animal Sustentável na Amazônia Ocidental (MESPA), instituição (UFAC). E-mail: michelinnedantas@bol.com.br.

${ }^{4}$ Engenheiro Agrônomo. Mestrando em Sanidade e Produção Animal Sustentável na Amazônia Ocidental (MESPA), instituição (UFAC). E-mail: adenhauer@hotmail.com.

${ }^{5}$ Ciências Biológicas. Mestre, Analista Ambiental do Instituto Brasileiro do Meio Ambiente (IBAMA). E-mail: elaineoliveira_ac@ yahoo.com.br.

${ }^{6}$ Médica Veterinária. Doutora, Docente do Centro de Ciências Biológicas e da Natureza da Universidade Federal do Acre (UFAC). E-mail: vania.rib@uol.com.br. 


\section{Introdução}

A bacia amazônica abrange a maior e mais diversificada parcela de floresta tropical contínua encontrado no mundo, que ocupa mais de seis milhões de quilômetros quadrados em nove países da América do Sul. Ela constitui o habitat de mais de 40.000 espécies de plantas, 427 de mamíferos, 1.294 de aves, 378 de répteis, 427 de anfíbios e mais de 3.000 espécies de peixes, representando cerca de $10 \%$ da biodiversidade do planeta (MITTERMEIER et al., 2003).

Estudos reforçam a importância do Acre em termos de representatividade biológica, pois cerca de $40 \%$ dos mamíferos do Brasil e 4,5\% dos mamíferos que ocorrem no mundo, existem no Acre. No caso das aves, 45,8\% das espécies existentes no Brasil e $8,5 \%$ das que existem no mundo, ocorrem no estado. De acordo com a Lista de Espécies de Vertebrados do Estado do Acre o grupo das aves apresenta a maior diversidade $(51,4 \%)$, seguido pelos peixes (21,6\%), mamíferos (13,6\%), anfíbios (7,4\%) e répteis $(6,0 \%)$, considerando os 1.498 registros como espécies (ACRE, 2010).

A exploração desordenada e predatória da biodiversidade expõe incessantemente os animais silvestres a vulnerações e a ameaças de extinção. Mesmo após o advento da Lei no 5.197/67 (Lei de Proteção à Fauna), e posteriormente, da Lei 9.605/98 (Lei de Crimes Ambientais), que torna crime a utilização da fauna para fins de consumo alimentar e criação, o problema persiste (DIAS JÚNIOR; CUNHA; DIAS, 2013).

O Instituto Brasileiro de Meio Ambiente e dos Recursos Renováveis (IBAMA) é o órgão federal responsável por executar as políticas públicas do meio ambiente e de fiscalização ambiental. Mantidos por este órgão a partir de suas superintendências estaduais ou através de parcerias com outras instituições públicas ou privadas, foram criados os Centros de Triagem de Animais Silvestres - CETAS (BRASIL, 2008). Atualmente, no Brasil existem 33
CETAS e desses, 30 (90,9\%) são de responsabilidade do IBAMA e três $(9,0 \%)$ de empresas privadas. Na região Amazônica funcionam sete $(21,2 \%)$ CETAS.

Os CETAS apresentam a finalidade de receber, triar, tratar, guardar e destinar os animais silvestres resgatados ou apreendidos pelos órgãos fiscalizadores, assim como, receber animais silvestres de particulares que fazem a entrega de forma voluntária e que eventualmente poderiam estar mantendo em cativeiros domésticos de forma irregular. Os animais podem ser soltos em áreas de preservação ou destinados para criadores cadastrados, como zoológicos e parques (CORDEIRO, 2009; FRANCO et al., 2012).

O recebimento dos animais nos CETAS pode ser classificado, de acordo com a procedência, em três formas distintas: a) apreensão, representada pelos animais decorrentes da ação fiscalizatória do IBAMA ou da Polícia Ambiental; b) recolhimento, resultado da captura de animais pelo IBAMA ou Polícia Ambiental; c) entrega voluntária, feita pelo cidadão que mantinha ilegalmente sob sua guarda animais silvestres (PAGANO et al., 2009).

O CETAS - ACRE é o único do estado e recebe animais de todos os municípios acreanos. A maioria desses animais é resgatada pelos bombeiros, atendendo as solicitações do Centro Integrado de Ensino e Pesquisa em Segurança Pública do Acre - CIOSP. No ato da recepção dos animais é preenchida uma ficha de recebimento do animal, que contém a sua classificação, o sexo quando possível, o tipo de entrada (apreensão, doação institucional, entrega voluntária ou nascimento), a faixa etária, condição física e de saúde. Nessa ficha é gerado o controle de everminação, suplementação de vitaminas e dados sobre a destinação e tipo de saída do animal. Os animais que são destinados ao cativeiro recebem um microchip, priorizando as aves que são mais difíceis para distinção de indivíduos.

O trabalho teve como objetivo realizar um levantamento das espécies de animais silvestres 
resgatados e posteriormente alojados no CETAS de Rio Branco - Acre de janeiro de 2010 a dezembro de 2014 e suas implicações ambientais conservacionistas.

\section{Material e Método}

O estudo descritivo foi desenvolvido através de análise de informações fornecidas pelo CETASACRE após consultas nos registros de entradas de animais silvestres, referente ao período de janeiro de 2010 a dezembro de 2014.

Foi seguido à classificação do Comitê Brasileiro de Registros Ornitológicos (2014) para a identificação das aves, do Catalogue of Life (2012) para os mamíferos e a Lista de Espécies da Herpetologia Brasileira para os répteis (BÉRNILS; COSTA, 2012) e para a confirmação das serpentes existentes no Acre foi consultado Silva, Souza e Bernarde (2010). Também foram consultados especialistas da área além de outras referências para identificação das espécies. Os animais foram identificados até o menor nível hierárquico possível.

As espécies ameaçadas foram catalogadas mediante consultas ao Livro Vermelho da Fauna Brasileira Ameaçada de Extinção (MACHADO; DRUMMOND; PAGLIA, 2008) e ao Anexo I da Lista Nacional Oficial de Espécies da Fauna Ameaçadas de Extinção - Portaria n ${ }^{\circ}$ 444/2014 (BRASIL, 2014).

Os dados foram organizados e analisados através de estatística descritiva e tabulados através do programa computacional Excel da Microsoft Office para a confecção de figuras e tabelas.

\section{Resultados}

O CETAS - ACRE recebeu entre os anos de 2010 e 2014 um total de 2.320 animais silvestres. Dentre estes, o grupo das aves foi predominante em relação aos demais, apresentando um total de 1.097 animais apreendidos (47,2\%), seguido pelo grupo dos répteis com 720 (31,0\%), mamíferos com $498(21,4 \%)$ e peixes com cinco animais $(0,2 \%)$. Dentre esses animais recepcionados no CETAS - ACRE, $0,6 \%$ consta na lista de animais em risco de extinção. O maior número de apreensões ocorreu no ano de 2012, com 559 animais recebidos no CETAS - ACRE, e o menor com 390 animais no ano de 2014 (Figura 1).

As espécies de aves mais apreendidas foram: Sporophila angolensis correspondendo a 46,0\%, Amazona ochrocephala com 10,2\% e o Brotogeris sanctithomae com 4,5\%. As demais espécies alojadas totalizaram 39,3\% (Tabela 1).

As espécies de mamíferos mais apreendidas foram: Didelphis marsupialis com 9,2\% animais alojados no CETAS, Cebus apella 7,6\%, Bradypus variegatus 6,8\%, Alouatta seniculus $5,6 \%$, Tamandua tetradactyla 5,6\% e finalmente o Dasypus novemcinctus com 5,4\%. As demais espécies totalizaram $59,8 \%$ dos alojamentos (Tabela 2).

As espécies de répteis mais apreendidas foram Chelonoidis denticulata (48,6\%), Boa constrictor $(26,5 \%)$ e Caiman crocodilus (10,0\%). As demais espécies totalizaram 14,9\% (Tabela 3).

No ano de 2010 foram recebidos cinco Electrophorus electricus (peixe elétrico), com nenhum alojamento de peixes nos demais anos. 
Figura 1 - Fauna silvestre recebida no Centro de Triagem de Animais Silvestres Acre de acordo com os grupos de animais, no período entre 2010 e 2014.

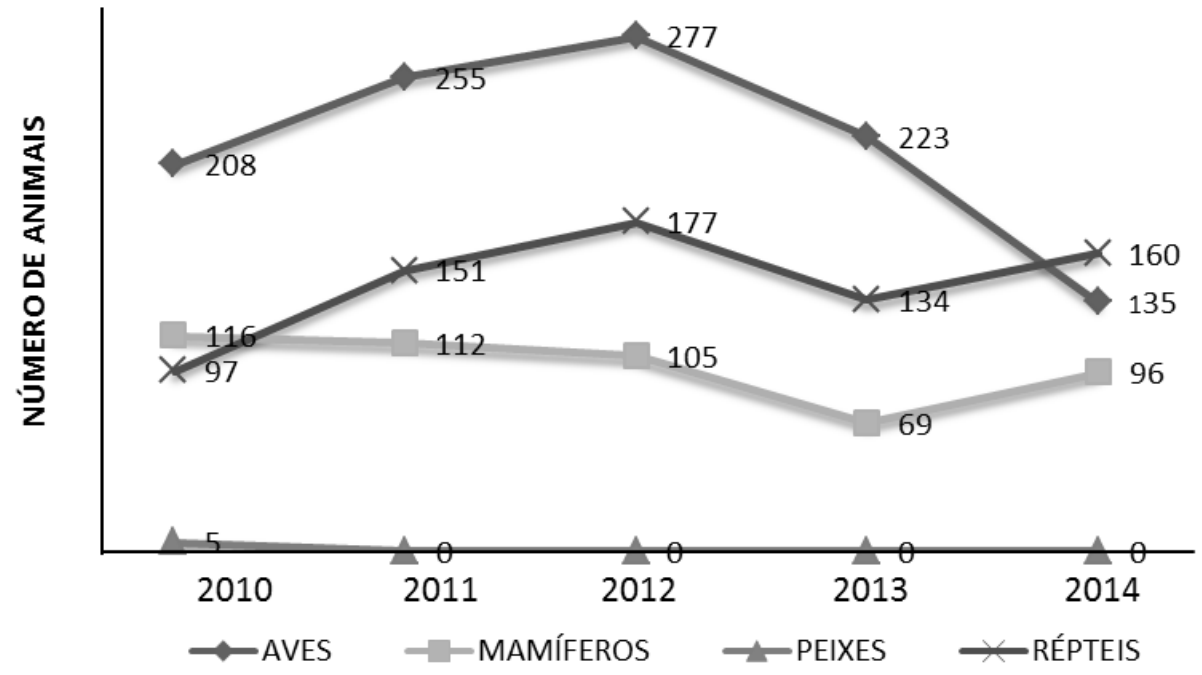

Fonte: IBAMA (2015).

Tabela 1 - Espécies de aves recebidas no Centro de Triagem de Animais Silvestres - Acre, no período entre 2010 e 2014. AE - ameaçado de extinção ( $\mathrm{N}=$ não, $\mathrm{S}=\operatorname{sim}, \mathrm{V}=$ vulnerável).

\section{AVES}

\begin{tabular}{lllllll} 
Espécie & Nome popular & \multicolumn{5}{c}{ Ano de recebimento } \\
& 2010 & 2011 & 2012 & 2013 & 2014
\end{tabular}

\begin{tabular}{lccccccccc}
\hline Alipiopsitta xanthops & Papagaio galego & 0 & 0 & 1 & 0 & 0 & 1 & 0,1 & $\mathrm{~N}$ \\
Amazona farinosa & Papagaio urubu & 1 & 4 & 7 & 3 & 2 & 17 & 1,6 & $\mathrm{~N}$ \\
Amazona festiva & Papagaio caboclo & 1 & 1 & 0 & 2 & 2 & 6 & 0,6 & $\mathrm{~N}$ \\
Amazona ochrocephala & Papagaio estrela & 19 & 43 & 15 & 23 & 12 & 112 & 10,2 & $\mathrm{~N}$ \\
Anas discors & Marreco & 0 & 0 & 1 & 0 & 0 & 1 & 0,1 & $\mathrm{~N}$ \\
Ara ararauna & Arara canindé & 0 & 2 & 0 & 3 & 0 & 5 & 0,5 & $\mathrm{~N}$ \\
Ara macao & Arara vermelha & 1 & 4 & 1 & 5 & 5 & 16 & 1,4 & $\mathrm{~N}$ \\
Ara severus & Maracanã & 2 & 2 & 0 & 0 & 0 & 4 & 0,4 & $\mathrm{~N}$ \\
$\begin{array}{l}\text { Aratinga } \\
\text { leucophthalmus }\end{array}$ & Periquito maracanã & 1 & 0 & 0 & 2 & 6 & 9 & 0,8 & $\mathrm{~N}$ \\
Aratinga weddellii & Periquito sujo & 5 & 2 & 6 & 1 & 9 & 23 & 2,1 & $\mathrm{~N}$ \\
Ardea alba & Garça & 1 & 0 & 0 & 0 & 0 & 1 & 0,1 & $\mathrm{~N}$ \\
Asio clamator & Coruja orelhuda & 14 & 13 & 8 & 3 & 2 & 40 & 3,7 & $\mathrm{~N}$ \\
$\begin{array}{l}\text { Brotogeris cyanoptera } \\
\text { Arostrata }\end{array}$ & Periquito estrela & 0 & 1 & 0 & 2 & 3 & 6 & 0,6 & $\mathrm{~N}$ \\
\hline
\end{tabular}




\begin{tabular}{|c|c|c|c|c|c|c|c|c|c|}
\hline $\begin{array}{l}\text { Brotogeris } \\
\text { sanctithomae }\end{array}$ & Periquito testinha & 6 & 13 & 18 & 9 & 3 & 49 & 4,5 & $\mathrm{~N}$ \\
\hline Buteo albonotatus & $\begin{array}{l}\text { Gavião de rabo } \\
\text { barrado }\end{array}$ & 0 & 0 & 0 & 0 & 1 & 1 & 0,1 & $\mathrm{~N}$ \\
\hline Buteo brachyurus & $\begin{array}{c}\text { Gavião de cauda } \\
\text { curta }\end{array}$ & 0 & 0 & 0 & 0 & 1 & 1 & 0,1 & $\mathrm{~N}$ \\
\hline Buteo magnirostris & Gavião caburé & 0 & 0 & 3 & 0 & 0 & 3 & 0,3 & $\mathrm{~N}$ \\
\hline Buteo nitidus & Gavião pedrês & 3 & 2 & 0 & 2 & 0 & 7 & 0,6 & $\mathrm{~N}$ \\
\hline Buteo sp. & Gavião & 2 & 0 & 0 & 0 & 1 & 3 & 0,3 & $\mathrm{~N}$ \\
\hline Butorides striatus & Socó & 0 & 2 & 0 & 0 & 0 & 2 & 0,2 & $\mathrm{~N}$ \\
\hline Cacicus cela & Japim & 0 & 0 & 1 & 0 & 1 & 2 & 0,2 & $\mathrm{~N}$ \\
\hline Cacicus sp. & Japim & 0 & 0 & 0 & 1 & 0 & 1 & 0,1 & $\mathrm{~N}$ \\
\hline Cairina moschata & Pato do mato & 7 & 0 & 0 & 0 & 0 & 7 & 0,6 & $\mathrm{~N}$ \\
\hline Carcara plancus & Gavião carcará & 0 & 0 & 0 & 1 & 0 & 1 & 0,1 & $\mathrm{~N}$ \\
\hline Casmerodius albus & Garça Branca & 0 & 0 & 1 & 0 & 1 & 2 & 0,2 & $\mathrm{~N}$ \\
\hline Chloroceryle aenea & $\begin{array}{l}\text { Martim-pescador- } \\
\text { anão }\end{array}$ & 0 & 1 & 0 & 0 & 0 & 1 & 0,1 & $\mathrm{~N}$ \\
\hline Chloroceryle inda & Martin pescador & 0 & 0 & 1 & 0 & 0 & 1 & 0,1 & $\mathrm{~N}$ \\
\hline Chloroceryle torquata & Martin pescador & 0 & 2 & 0 & 0 & 0 & 2 & 0,2 & $\mathrm{~N}$ \\
\hline $\begin{array}{l}\text { Cochlearius } \\
\text { cochlearius }\end{array}$ & Arapapá & 0 & 0 & 0 & 1 & 0 & 1 & 0,1 & $\mathrm{~N}$ \\
\hline Columba livia & Pombo comum & 0 & 3 & 3 & 3 & 2 & 11 & 1,0 & $\mathrm{~N}$ \\
\hline Columbina talpacoti & Rolinha roxa & 0 & 1 & 2 & 0 & 1 & 4 & 0,4 & $\mathrm{~N}$ \\
\hline Coragyps atratus & Urubu & 0 & 2 & 6 & 1 & 0 & 9 & 0,8 & $\mathrm{~N}$ \\
\hline Crypturellus sp. & Nambú & 0 & 0 & 2 & 0 & 0 & 2 & 0,2 & V \\
\hline Crotophaga ani & Anum preto & 0 & 0 & 1 & 1 & 0 & 2 & 0,2 & $\mathrm{~N}$ \\
\hline Daptrius ater & Gavião preto & 0 & 0 & 1 & 0 & 0 & 1 & 0,1 & $\mathrm{~N}$ \\
\hline Diopsittaca nobilis & Maracanã & 3 & 0 & 0 & 0 & 0 & 3 & 0,3 & $\mathrm{~N}$ \\
\hline Egretta caerulea & Garça azul & 0 & 0 & 1 & 0 & 0 & 1 & 0,1 & $\mathrm{~N}$ \\
\hline Elanoides forficatus & Gavião tesoura & 0 & 0 & 1 & 0 & 0 & 1 & 0,1 & $\mathrm{~N}$ \\
\hline Estrilda astrild & Bico-de-lacre & 0 & 1 & 0 & 2 & 0 & 3 & 0,3 & $\mathrm{~N}$ \\
\hline Euphonia violacea & $\begin{array}{l}\text { Gaturamo } \\
\text { verdadeiro }\end{array}$ & 2 & 0 & 0 & 0 & 0 & 2 & 0,2 & $\mathrm{~N}$ \\
\hline Falco deiroleucus & $\begin{array}{l}\text { Falcão de peito } \\
\text { laranja }\end{array}$ & 0 & 0 & 0 & 0 & 2 & 2 & 0,2 & $\mathrm{~N}$ \\
\hline Geotrygon sp. & Rolinha pariri & 0 & 0 & 1 & 0 & 0 & 1 & 0,1 & $\mathrm{~N}$ \\
\hline
\end{tabular}


Geotrygon violacea

Geranoaetus

albicaudatus

Glaucidium

brasilianum

Glaucidium hardyi

Harpagus bidentatus

Harpia harpyja

Heliornes fulica

Hydropsalis albicollis

Hydropsalis torquata

Ictinia plumbea

Jacana jacana

Luscinia megarhynchos

Macropsalis sp.

Megascops choliba

Micrastur ruficollis

Mitu tuberosum

Nemosia pileata

Nyctibius aethereus

Nyctibius grandis

Nyctibius griseus

Nyctiprogne leucopyga

Opisthocomus hoazin

Orthopsittaca manilata

Oryzoborus

maximiliani

Patagioenas

cayennensis

Penelope jacquacu

Pionites leucogaster

Pionus menstrus

Pitangus sulphuratus

Porphyrula martinica
Juriti

Gavião de rabo branco

$\begin{array}{llllllllll}\text { Coruja buraqueira } & 0 & 1 & 6 & 0 & 1 & 8 & 0,7 & \text { N }\end{array}$

Coruja caburé da Amazônia

Gavião-rapina

Gavião-real

Pássaro picaparra

Bacurau

Bacurau tesoura

Sovi

Jaçanã

Rouxinol

Bacurau

Coruja-do-Mato

Gavião caburé

Mutum

Saíra

Mãe da Lua

Mãe da lua gigante

Urutau

Bacurau

Cigana

Arara maracanã

Bicudo

Pomba galega

Jacu, jacupemba

Marianinha laranja

Curica-de-cabeçaroxa

Bem-te-vi

Frango d'água azul

200

0

2

$0,2 \quad \mathrm{~N}$

$\begin{array}{llllllll}0 & 0 & 0 & 1 & 0 & 1 & 0,1 & \mathrm{~N}\end{array}$

(0)

$\begin{array}{llllllll}0 & 0 & 1 & 0 & 0 & 1 & 0,1 & \mathrm{~N}\end{array}$

\begin{tabular}{|c|c|c|c|c|c|c|c|c|c|}
\hline Megascops choliba & Coruja-do-Mato & 0 & 4 & 8 & 2 & 5 & 19 & 1,7 & $\mathrm{~N}$ \\
\hline Micrastur ruficollis & Gavião caburé & 0 & 0 & 0 & 1 & 0 & 1 & 0,1 & $\mathrm{~N}$ \\
\hline Mitu tuberosum & Mutum & 0 & 0 & 0 & 1 & 0 & 1 & 0,1 & $\mathrm{~N}$ \\
\hline Nemosia pileata & Saíra & 0 & 0 & 1 & 0 & 0 & 1 & 0,1 & $\mathrm{~N}$ \\
\hline Nyctibius aethereus & Mãe da Lua & 0 & 0 & 0 & 3 & 0 & 3 & 0,3 & $\mathrm{~S}$ \\
\hline Nyctibius grandis & Mãe da lua gigante & 0 & 2 & 6 & 0 & 1 & 9 & 0,8 & $\mathrm{~N}$ \\
\hline Nyctibius griseus & Urutau & 0 & 0 & 0 & 0 & 1 & 1 & 0,1 & $\mathrm{~N}$ \\
\hline Nyctiprogne leucopyga & Bacurau & 1 & 0 & 0 & 0 & 0 & 1 & 0,1 & $\mathrm{~N}$ \\
\hline Opisthocomus hoazin & Cigana & 0 & 0 & 0 & 0 & 1 & 1 & 0,1 & $\mathrm{~N}$ \\
\hline Orthopsittaca manilata & Arara maracanã & 0 & 0 & 2 & 1 & 0 & 3 & 0,3 & $\mathrm{~N}$ \\
\hline $\begin{array}{l}\text { Oryzoborus } \\
\text { maximiliani }\end{array}$ & Bicudo & 2 & 0 & 0 & 0 & 0 & 2 & 0,2 & $\mathrm{~N}$ \\
\hline $\begin{array}{l}\text { Patagioenas } \\
\text { cayennensis }\end{array}$ & Pomba galega & 0 & 0 & 0 & 1 & 0 & 1 & 0,1 & $\mathrm{~N}$ \\
\hline Penelope jacquacu & Jacu, jacupemba & 0 & 1 & 1 & 1 & 1 & 4 & 0,4 & $\mathrm{~N}$ \\
\hline Pionites leucogaster & Marianinha laranja & 0 & 0 & 2 & 0 & 0 & 2 & 0,2 & $\mathrm{~N}$ \\
\hline Pionus menstrus & $\begin{array}{c}\text { Curica-de-cabeça- } \\
\text { roxa }\end{array}$ & 5 & 10 & 7 & 2 & 2 & 26 & 2,4 & $\mathrm{~N}$ \\
\hline Pitangus sulphuratus & Bem-te-vi & 0 & 1 & 1 & 1 & 1 & 4 & 0,4 & $\mathrm{~N}$ \\
\hline Porphyrula martinica & Frango d'água azul & 3 & 5 & 7 & 9 & 3 & 27 & 2,5 & $\mathrm{~N}$ \\
\hline
\end{tabular}


Porphyrio flavirostris

Primolius couloni

Psophia viridis

Pteroglossus castanotis

Pteroglossus sp.

Pygochelidon

cyanoleuca

Ramphocelus carbo

Rupornis magnirostris

Selenidera

maculirostris

Serinus sp.

Spizaetus ornatus

Sporophila angolensis

Sporophila

castaneiventris

Sporophila lineola

Sporophila sp.

Strix huhula

Tangara cayana

Tangara cyanoptera

Tangara episcopus

Tangara palmarum

Tigrisoma fasciatum

Tigrisoma lineatum

Turdus amaurochalinus

Turdus rufiventris

Turdus sp.

Tyto alba

Vanellus chilenses

Volatinia jacarina
Frango d'água

$$
\text { pequeno }
$$

Maracanã couloni

Jacamin

Araçari castanhos

Araçari

2

(1)

0

- -2

$2 \quad 0,2 \quad \mathrm{~N}$

Andorinha

Pipira

Gavião-carijó

Araçari

Canário belga

Gavião de penacho

Curió

110

Caboclinho de peito castanho

Coleirinha

Coruja preta

Saíra amarela

Sanhaço-de-

encontro-azul

Sanhaço

Sanhaçu do coqueiro

Socó

Socó-boi

Sabiá

Sabiá

Sabiá

Coruja suindara

Quero-quero

Tiziu

$\begin{array}{ll}0 & 0 \\ 2 & 0\end{array}$

1

0

0

$10,1 \quad \mathrm{~N}$

$\begin{array}{ll}2 & 0 \\ 0 & 0\end{array}$

$\begin{array}{lll}0 & 0 & 2 \\ 4 & 1 & 0\end{array}$

0

0

2

$0,2 \quad \mathrm{~N}$

$\begin{array}{llllllll}0 & 0 & 0 & 1 & 0 & 1 & 0,1 & \mathrm{~N}\end{array}$

$\begin{array}{llllllll}0 & 0 & 1 & 0 & 0 & 1 & 0,1 & \mathrm{~N}\end{array}$

$\begin{array}{llllllll}1 & 5 & 3 & 6 & 8 & 23 & 2,1 & \mathrm{~N}\end{array}$

$\begin{array}{llllllll}0 & 0 & 1 & 0 & 0 & 1 & 0,1 & \mathrm{~N}\end{array}$


Tabela 2 - Espécies de mamíferos recebidas no Centro de Triagem de Animais Silvestres - Acre, no período entre 2010 e 2014. AE - Ameaçado de extinção ( $\mathrm{N}=$ não, $\mathrm{S}=\operatorname{sim}, \mathrm{V}=$ vulnerável).

\section{MAMÍFEROS}

$\begin{array}{lllllllll}\text { Espécie } & \text { Nome popular } & & \text { Ano de recebimento } & \text { Total } & \text { AE }\end{array}$

\begin{tabular}{|c|c|c|c|c|c|c|c|c|c|}
\hline Agouti paca & Paca & 0 & 1 & 0 & 0 & 0 & 1 & 0,2 & $\mathrm{~N}$ \\
\hline Alouatta seniculus & Macaco guariba & 6 & 5 & 8 & 5 & 4 & 28 & 5,6 & $\mathrm{~N}$ \\
\hline Aotus nigriceps & Macaco da noite & 2 & 11 & 2 & 1 & 0 & 16 & 3,2 & $\mathrm{~N}$ \\
\hline Ateles paniscus & Macaco-aranha & 0 & 1 & 1 & 0 & 0 & 2 & 0,4 & $\mathrm{~N}$ \\
\hline Ateles paniscus chamek & Macaco-aranha & 0 & 0 & 0 & 0 & 2 & 2 & 0,4 & $\mathrm{~N}$ \\
\hline Bradypus tridactylus & Preguiça de bentinho & 2 & 0 & 0 & 1 & 0 & 3 & 0,6 & $\mathrm{~N}$ \\
\hline Bradypus variegatus & Preguiça comum & 14 & 2 & 6 & 3 & 9 & 34 & 6,8 & $\mathrm{~N}$ \\
\hline Callicebus cupreus & Macaco zog zog & 5 & 3 & 0 & 0 & 0 & 8 & 1,6 & $\mathrm{~N}$ \\
\hline Callicebus sp. & Macaco zog zog & 0 & 0 & 0 & 0 & 1 & 1 & 0,2 & $\mathrm{~S}$ \\
\hline Callimico goeldii & Taboqueiro & 0 & 0 & 1 & 1 & 0 & 2 & 0,4 & $\mathrm{~N}$ \\
\hline Cebuella pygmae & Leãozinho da taboca & 5 & 0 & 2 & 1 & 0 & 8 & 1,6 & $\mathrm{~N}$ \\
\hline Cebus albifrons & Macaco cairara & 1 & 5 & 7 & 0 & 2 & 15 & 3,0 & $\mathrm{~N}$ \\
\hline Cebus apella & Macaco prego & 14 & 8 & 5 & 7 & 4 & 38 & 7,6 & $\mathrm{~N}$ \\
\hline Choloepus hoffmanni & Pregiça hoffmanni & 0 & 3 & 0 & 1 & 0 & 4 & 0,8 & $\mathrm{~N}$ \\
\hline Choloepus didactylus & Pregiça real & 0 & 0 & 0 & 0 & 3 & 3 & 0,6 & $\mathrm{~N}$ \\
\hline Choloepus sp. & Pregiça real & 0 & 0 & 0 & 1 & 0 & 1 & 0,2 & $\mathrm{~N}$ \\
\hline Cyclopes didactylus & Tamanduaí & 7 & 4 & 2 & 3 & 4 & 20 & 4,0 & $\mathrm{~N}$ \\
\hline Coendou prehensilis & Porco-espinho & 3 & 2 & 0 & 0 & 9 & 14 & 2,8 & $\mathrm{~N}$ \\
\hline Cuniculus paca & Paca & 0 & 0 & 3 & 2 & 1 & 6 & 1,2 & $\mathrm{~N}$ \\
\hline Dasyprocta fuliginosa & Cutia & 3 & 5 & 5 & 2 & 2 & 17 & 3,4 & $\mathrm{~N}$ \\
\hline Dasypus novemcinctus & Tatu-galinha & 8 & 3 & 7 & 6 & 3 & 27 & 5,4 & $\mathrm{~N}$ \\
\hline Dasypus sp. & Tatu & 0 & 1 & 0 & 0 & 0 & 1 & 0,2 & $\mathrm{~N}$ \\
\hline Didelphis albiventris & Mucura & 8 & 0 & 0 & 0 & 0 & 8 & 1,6 & $\mathrm{~N}$ \\
\hline Didelphis marsupialis & Mucura & 0 & 10 & 20 & 6 & 10 & 46 & 9,2 & $\mathrm{~N}$ \\
\hline Dinomys branickii & Paca de rabo & 2 & 1 & 3 & 2 & 5 & 13 & 2,6 & $\mathrm{~N}$ \\
\hline Eira barbara & Irara & 0 & 0 & 0 & 1 & 0 & 1 & 0,2 & $\mathrm{~N}$ \\
\hline
\end{tabular}




\begin{tabular}{|c|c|c|c|c|c|c|c|c|c|}
\hline Galictis vittata & Furão da Amazônia & 2 & 1 & 0 & 0 & 0 & 3 & 0,6 & $\mathrm{~N}$ \\
\hline Hydrochaeris hydrochaeris & Capivara & 4 & 2 & 2 & 3 & 10 & 21 & 4,2 & $\mathrm{~N}$ \\
\hline Inia geoffrensis & Boto rosa & 1 & 0 & 0 & 0 & 0 & 1 & 0,2 & $\mathrm{~N}$ \\
\hline Lagothrix lagothicha & Macaco barrigudo & 2 & 5 & 0 & 0 & 1 & 8 & 1,6 & $\mathrm{~V}$ \\
\hline Leopardus pardalis & Jaguatirica & 2 & 4 & 2 & 4 & 1 & 13 & 2,6 & $\mathrm{~N}$ \\
\hline Leopardus wiedii & Gato-maracajá & 0 & 1 & 0 & 1 & 0 & 2 & 0,4 & $\mathrm{~V}$ \\
\hline Lutra longicaudis & Lontra & 0 & 0 & 2 & 0 & 0 & 2 & 0,4 & $\mathrm{~N}$ \\
\hline Mazama americana & Veado-mateiro & 0 & 2 & 0 & 0 & 0 & 2 & 0,4 & $\mathrm{~N}$ \\
\hline Mazama nemorivaga & Veado roxo & 0 & 0 & 1 & 0 & 0 & 1 & 0,2 & $\mathrm{~N}$ \\
\hline Não identificado & Rato selvagem & 1 & 0 & 0 & 0 & 0 & 1 & 0,2 & $\mathrm{~N}$ \\
\hline Nasua nasua & Quati & 3 & 3 & 1 & 1 & 6 & 14 & 2,8 & $\mathrm{~N}$ \\
\hline Panthera onca & Onça pintada & 0 & 0 & 1 & 0 & & 1 & 0,2 & $\mathrm{~V}$ \\
\hline Pecari tajacu & Porco do mato & 3 & 1 & 0 & 0 & 3 & 7 & 1,4 & $\mathrm{~N}$ \\
\hline Pithecia irrorata & Macaco-parauacu & 0 & 1 & 2 & 1 & 0 & 4 & 0,8 & $\mathrm{~N}$ \\
\hline Potos flavus & Jupará & 0 & 0 & 1 & 0 & 0 & 1 & 0,2 & $\mathrm{~N}$ \\
\hline Procyon sp. & Guaxinim & 1 & 0 & 0 & 0 & 0 & 1 & 0,2 & $\mathrm{~N}$ \\
\hline Procyon cancryvorus & Guaxinim & 0 & 3 & 0 & 0 & 1 & 4 & 0,8 & $\mathrm{~N}$ \\
\hline Puma concolor & Onça parda & 0 & 0 & 0 & 0 & 2 & 2 & 0,4 & $\mathrm{~V}$ \\
\hline Saguinus fuscicollis & Macaco soim & 4 & 7 & 3 & 4 & 0 & 18 & 3,6 & $\mathrm{~N}$ \\
\hline Saguinus imperator & Macaco bigodeiro & 3 & 2 & 1 & 3 & 2 & 11 & 2,2 & $\mathrm{~N}$ \\
\hline Saguinus labiatus & Sagui labiatus & 0 & 0 & 1 & 0 & 0 & 1 & 0,2 & $\mathrm{~N}$ \\
\hline Saguinus sp. & Macaco soim & 0 & 0 & 0 & 0 & 3 & 3 & 0,6 & $\mathrm{~S}$ \\
\hline Saimiri boliviensis & Macaco de cheiro & 0 & 6 & 2 & 2 & 2 & 12 & 2,4 & $\mathrm{~N}$ \\
\hline Saimiri sciureus & Macaco de cheiro & 6 & 0 & 0 & 0 & 0 & 6 & 1,2 & $\mathrm{~N}$ \\
\hline Sciurus aestuans & Quatipuru vermelho & 1 & 0 & 0 & 0 & 0 & 1 & 0,2 & $\mathrm{~N}$ \\
\hline Tamandua tetradactyla & Mambira & 3 & 6 & 8 & 5 & 6 & 28 & 5,6 & $\mathrm{~N}$ \\
\hline Tapirus terrestris & Anta & 0 & 0 & 1 & 0 & 0 & 1 & 0,2 & $\mathrm{~V}$ \\
\hline Tayassu pecari & Queixada & 0 & 3 & 0 & 0 & 0 & 3 & 0,6 & $\mathrm{~V}$ \\
\hline Tayassu tajacu & Porco do mato & 0 & 0 & 5 & 2 & 0 & 7 & 1,4 & $\mathrm{~N}$ \\
\hline TOT & & 116 & 112 & 105 & 69 & 96 & 498 & 100 & \\
\hline
\end{tabular}

Fonte: IBAMA (2015). 
Tabela 3 - Espécies de répteis recebidas no Centro de Triagem de Animais Silvestres - Acre, no período entre 2010 e 2014. AE - ameaçado de extinção ( $N$ = não, $S=$ sim, $V=$ vulnerável).

\section{RÉPTEIS}

\begin{tabular}{|c|c|c|c|c|c|c|c|c|c|}
\hline \multirow{2}{*}{ Espécie } & \multirow{2}{*}{ Nome popular } & \multicolumn{5}{|c|}{ Ano de recebimento } & \multirow{2}{*}{ Total } & \multirow{2}{*}{$\%$} & \multirow{2}{*}{$\mathrm{AE}$} \\
\hline & & 2010 & 2011 & 2012 & 2013 & 2014 & & & \\
\hline Anilius scytale & Falsa-coral & 0 & 0 & 1 & 0 & 0 & 1 & 0,1 & $\mathrm{~N}$ \\
\hline Boa constrictor & Jiboia & 42 & 37 & 40 & 32 & 40 & 191 & 26,5 & $\mathrm{~N}$ \\
\hline $\begin{array}{l}\text { Bothrocophias } \\
\text { hyoprora }\end{array}$ & Jararaca bicuda & 0 & 0 & 1 & 0 & 0 & 1 & 0,1 & $\mathrm{~N}$ \\
\hline Bothrops atrox & Cobra jararaca & 1 & 0 & 0 & 1 & 0 & 2 & 0,2 & $\mathrm{~N}$ \\
\hline Caiman crocodilus & Jacaré-tinga & 8 & 21 & 24 & 10 & 9 & 72 & 10,0 & $\mathrm{~N}$ \\
\hline Chelus fimbriatus & Matamatá & 0 & 0 & 0 & 1 & 0 & 1 & 0,1 & $\mathrm{~N}$ \\
\hline $\begin{array}{l}\text { Chelonoidis } \\
\text { denticulata }\end{array}$ & Jabuti-tinga & 27 & 74 & 90 & 77 & 82 & 350 & 48,6 & $\mathrm{~N}$ \\
\hline $\begin{array}{l}\text { Chironius } \\
\text { bicarinatus }\end{array}$ & Cobra cipó & 1 & 0 & 0 & 0 & 0 & 1 & 0,1 & $\mathrm{~N}$ \\
\hline Clelia clelia & Mussurana & 0 & 1 & 1 & 0 & 0 & 2 & 0,2 & $\mathrm{~N}$ \\
\hline Corallus hortulanu & Suaçuboia & 0 & 0 & 0 & 1 & 0 & 1 & 0,1 & $\mathrm{~N}$ \\
\hline Dracaena guianensis & $\begin{array}{l}\text { Lagarto- } \\
\text { jacarerana }\end{array}$ & 0 & 1 & 0 & 0 & 0 & 1 & 0,1 & $\mathrm{~N}$ \\
\hline Epicrates cenchria & Salamantra & 0 & 0 & 1 & 2 & 0 & 3 & 0,4 & $\mathrm{~N}$ \\
\hline Eunectes murinus & Sucuri & 8 & 2 & 5 & 3 & 7 & 25 & 3,5 & $\mathrm{~N}$ \\
\hline Geoframus sp. & Cágado & 0 & 0 & 0 & 0 & 1 & 1 & 0,1 & $\mathrm{~N}$ \\
\hline Iguana iguana & Iguana & 0 & 0 & 0 & 1 & 1 & 2 & 0,3 & $\mathrm{~N}$ \\
\hline $\begin{array}{l}\text { Kinosternon } \\
\text { scorpioides }\end{array}$ & Muçuã & 2 & 3 & 0 & 0 & 4 & 9 & 1,3 & $\mathrm{~N}$ \\
\hline Lachesis muta & $\begin{array}{l}\text { Surucucu-pico-de- } \\
\text { jaca }\end{array}$ & 0 & 2 & 1 & 0 & 0 & 3 & 0,4 & $\mathrm{~N}$ \\
\hline Mesoclemmys gibba & Cágado & 0 & 0 & 1 & 0 & 0 & 1 & 0,1 & $\mathrm{~N}$ \\
\hline $\begin{array}{l}\text { Oxyrhopus } \\
\text { melanogenys }\end{array}$ & Falsa coral & 0 & 0 & 1 & 0 & 0 & 1 & 0,1 & $\mathrm{~N}$ \\
\hline $\begin{array}{l}\text { Paleosuchus } \\
\text { palpebrosus }\end{array}$ & Jacaré-coroa & 0 & 1 & 2 & 0 & 0 & 3 & 0,4 & $\mathrm{~N}$ \\
\hline $\begin{array}{l}\text { Paleosuchus } \\
\text { trigonatus }\end{array}$ & Jacaré-coroa & 0 & 0 & 0 & 0 & 2 & 2 & 0,3 & $\mathrm{~N}$ \\
\hline Platemys platycephala & Jabuti machado & 0 & 2 & 0 & 1 & 0 & 3 & 0,4 & $\mathrm{~N}$ \\
\hline Phrynops geoffroanus & Cágado & 0 & 1 & 0 & 0 & 1 & 2 & 0,3 & $\mathrm{~N}$ \\
\hline
\end{tabular}




\begin{tabular}{|c|c|c|c|c|c|c|c|c|c|}
\hline Phrynops sp. & $\begin{array}{l}\text { Cágado de } \\
\text { barbicha }\end{array}$ & 0 & 0 & 0 & 1 & 0 & 1 & 0,1 & $\mathrm{~N}$ \\
\hline Podocnemis expansa & $\begin{array}{l}\text { Tartaruga da } \\
\text { Amazônia }\end{array}$ & 4 & 0 & 1 & 1 & 7 & 13 & 1,8 & $\mathrm{~N}$ \\
\hline Podocnemis unifilis & Tracajá & 2 & 4 & 6 & 3 & 2 & 17 & 2,4 & $\mathrm{~N}$ \\
\hline Spilotes pullatus & Caninana & 1 & 0 & 2 & 1 & 3 & 7 & 1,0 & $\mathrm{~N}$ \\
\hline $\begin{array}{l}\text { Trachemys scripta } \\
\text { elegans }\end{array}$ & Tigre d’água & 0 & 0 & 0 & 0 & 1 & 1 & 0,1 & $\mathrm{~N}$ \\
\hline Tupinambis sp & Lagarto teiú & 0 & 2 & 0 & 0 & 0 & 2 & 0,3 & $\mathrm{~N}$ \\
\hline Xenodon sp. & Boipeva & 1 & 0 & 0 & 0 & 0 & 1 & 0,1 & $\mathrm{~N}$ \\
\hline \multicolumn{2}{|c|}{ Total } & 97 & 151 & 177 & 135 & 160 & 720 & 100 & \\
\hline
\end{tabular}

Fonte: IBAMA (2015).

\section{Discussão}

O grupo das aves predominou em relação aos demais, isso se dá pela beleza e o canto que atraem a população que os desejam como animais de estimação. Além disso, são muito apreciados por grupos de colecionadores, pois possuem extraordinária beleza que encantam os amantes de pássaros de gaiola (BARBOSA; NÓBREGA; ALVES, 2010).

Os dados confirmam os resultados obtidos em âmbito nacional, nos quais pesquisas afirmam que no Brasil a maior parte da fauna apreendida é composta por aves (BASTOS et al., 2008; FREITAS et. al., 2015; MOURA et al., 2012; RENCTAS, 2001; SILVA; LIMA, 2014). Portanto, percebe-se a preferência do tráfico por esses animais que, muitas vezes, apresentam o propósito da estimação por colecionadores e populares.

O curió (Sporophila angolensis) é o pássaro mais alojado, pois possui quantidade bem expressiva em relação aos demais pássaros e animais na região, de acordo com o número de apreensões do CETAS ACRE. Um dos motivos que levam essa ave a ser tão procurada é o seu canto característico, que atraem traficantes e populares que os querem para serem usados como animal de estimação. Apesar da grande procura por essa ave, a mesma ainda não consta nas listas entre as espécies ameaçadas de extinção (MACHADO; DRUMMOND; PAGLIA, 2008).

Alguns animais recebidos no CETAS constam entre as espécies tidas como vulneráveis e ameaçadas de extinção (IBAMA, 2008). Desta forma, estão em vulnerabilidade as espécies: Crypturellus sp., Harpia harpyja, Sporophila sp., Strix huhula, Tigrisoma fasciatum, Lagothrix lagothicha, Leopardus wiedii, Panthera onca, Puma concolor, Tapirus terrestres e Tayassu pecari. Está em perigo de extinção a espécie de Nyctibius aethereus e criticamente em perigo de extinção as espécies: Callicebus sp., Saguinus sp.

Acredita-se que a dificuldade de fiscalização enfrentada pelos profissionais da área e o pouco investimento em combate a essa prática, torna o tráfico de fauna silvestre cada vez mais promissor, tendo em vista à dificuldade de se cumprir a lei pela falta de apreensão do infrator (RENCTAS, 2001). De forma geral, há necessidade de planejamento adequado, sistematizado e recursos suficientes para o êxito das operações contra a retirada dos animais silvestres do seu habitat natural de forma ilegal (BASTOS et al., 2008).

A fauna silvestre, além da importância científica, social, estética e econômica, é fundamental para a sustentabilidade dos ecossistemas (PORTAL BRASIL, 2012) e sua retirada do habitat natural, 
poderá afetar a polinização e a dispersão de propágulos, e consequentemente, a manutenção das florestas. Os primatas e as aves possuem grande importância no processo de dispersão (PIZO, 2003).

Grande parte das doenças infecciosas emergentes é representada por patógenos causadores de zoonoses que representam $60,3 \%$ do total de doenças infecciosas emergentes e, do total de zoonoses, 71,8\% têm origem em animais silvestres (JONES et al., 2008).

Dessa forma, o tráfico de animais silvestres e seus produtos, podem contribuir com a extinção de espécies além de oferecer riscos à saúde pública. É sugerida uma maior fiscalização para evitar a manutenção desses animais principalmente em cativeiros domésticos, com o intuito de evitar a exposição das pessoas às suas possíveis zoonoses.

\section{Conclusão}

Conclui-se que apesar das fiscalizações do policiamento ambiental e das ações do IBAMA contra o tráfico de animais silvestres, os animais considerados em risco de extinção e vulneráveis ainda continuam sendo explorados e retirados do seu habitat natural. Verificou-se que as aves são os animais silvestres mais predados considerando o número de indivíduos dessa classe recebidos pelo Centro de Triagem. Dessa forma, ações de educação ambiental devem ser efetivadas por meio de órgãos governamentais e não governamentais da sociedade civil, para conscientizar a população da importância de preservar os animais na natureza, e que, se estes vierem a compor um cenário doméstico, que sejam procedentes de criatórios legalizados onde a saúde animal é controlada por profissionais.

\section{Referências}

ACRE. Secretaria de Estado de Meio Ambiente. Programa Estadual de Zoneamento EcológicoEconômico do Acre. Recursos naturais: biodiversidade e ambientes do Acre: zoneamento ecológico-econômico fase II. Rio Branco, 2010.
BARBOSA, J. A. A.; NOBREGA, V. A.; ALVES, R. R. N. Aspectos da caça e comércio ilegal da avifauna silvestre por populações tradicionais do semi-árido paraibano. Revista de Biologia e Ciências da Terra, Aracaju, v. 10, n. 2, p. 39-49, 2010.

BASTOS, L. F.; LUZ, V. L. F.; REIS, I. J.; SOUZA, V. L. Apreensão de espécimes da fauna silvestre em Goiás: situação e destinação. Revista de Biologia Neotropical, Goiânia, v. 5, n. 2, p. 51-63, 2008.

BÉRNILS R. S.; COSTA, H. C. Répteis brasileiros: lista de espécies. 2012. Disponível em: <http:// www.sbherpetologia.org.br/lista_repteis/ ListaRepteis30Setembro2012PORTUGŪES.pdf.>. Acesso em: 5 nov. 2015.

BRASIL. Ministério do Meio Ambiente. Instrução Normativa n. 169, de 20 de fevereiro de 2008. Disponível em: <http://www.mma.gov.br/port/ conama/legiabre.cfm?codlegi=585. $>$. Acesso em: 2 jun. 2015.

BRASIL. Ministério do Meio Ambiente. Portaria n. 444, de 17 de dezembro de 2014. Lista Nacional das Espécies da Fauna Brasileira Ameaçadas de Extinção. 2014. Disponível em: <http://www. mma.gov.br/destaques/itemlist/category/51especies-ameacadas-de-extincao $>$. Acesso em: 2 jun. 2015.

CATALOGUE OF LIFE. Annual checklist: indexing the world's species. 2012. Disponível em: $<$ http:// www.catalogueoflife.org/search.php $>$. Acesso em: 22 jun. 2015.

COMITÊ BRASILEIRO DE REGISTROS ORNITOLÓGICOS - CBRO. Aves do Brasil, 2014. Disponível em: $<$ http://www.taxeus.com. br/listamaisinformacoes/2582>. Acesso em: 11 nov. 2015.

CORDEIRO, A. L. L. Centro de Triagem de Animais Silvestres - CETAS. Bastidores das destinações dos animais protocolos normativos. In: ANAIS DO IX CONGRESSO DE ECOLOGIA DO BRASIL, 9., 2009, São Lourenço. Anais... São Lourenço: SEB, 2009. Disponível em: <http://www.seb-ecologia. org.br/2009/resumos_ixceb/1450.pdf>. Acesso em: 5 ago. 2015. 
DIAS JÚNIOR, M. B. F; CUNHA, H. F. A.; DIAS, T. C. A. C. Análise da destinação da fauna silvestre apreendida no Estado do Amapá, Brasil. Planeta Amazônia: Revista Internacional de Direito Ambiental e Políticas Públicas, Macapá, n. 5, p. 23-36, 2013.

FRANCO, M. R.; CÂMARA, F. M.; ROCHA, D. C. C.; SOUZA, R. M.; OLIVEIRA, N. J. F. Animais silvestres apreendidos no período de 2002 a 2007 na macrorregião de Montes Claros, Minas Gerais. Enciclopédia Biosfera, Goiânia, v. 8, n. 14, p. 10071018, 2012.

FREITAS, A. C. P.; OVIEDO-PASTRANA, M. E.; VILELA, D. A. R.; PEREIRA, P. L. L.; LOUREIRO, L. O. C.; HADDAD, J. P. A.; MARTINS, N. R. S.; SOARES, D. F. M. Diagnóstico de animais ilegais recebidos no centro de triagem de animais silvestres de Belo Horizonte, Estado de Minas Gerais, no ano de 2011. Ciência Rural, Santa Maria, v. 45, n. 1, p. 163-170, jan. 2015.

IBAMA. Instituto Brasileiro do Meio Ambiente e dos Recursos Naturais Renováveis. Unidade Ibama-AC. Disponível em: <http://www.ibama. gov.br/acesso-a-informacao/unidade-ibama-ac $>$. Acesso em: 15 jun. 2015.

IBAMA. Instituto Brasileiro do Meio Ambiente e dos Recursos Naturais Renováveis. Instrução Normativa $n^{\circ} 179$, de 25 de junho 2008. Disponível em: <https://www.mprs.mp.br/areas/gapp/arquivos/ instrucao_normativa_ibama_179_de_2008.pdf $>$. Acesso em: Acesso em: 15 jun. $20 \overline{15}$.

JONES, K. E.; PATEL, N. G.; LEVY, M. A.; STOREYGARD, A.; BALK, D.; GITTLEMAN, J. L.; DASZAK, P. Global trends in emerging infectious diseases. Nature, London, v. 451, n. 7181, p. 990-993, 2008.

MACHADO, A. B. M.; DRUMMOND, G. M.; PAGLIA, A. P. Livro vermelho da fauna brasileira ameaçada de extinção. Brasília: Fundação Biodiversitas, 2008.

MITTERMEIER, R. A.; MITTERMEIER, C. G.; BROOKS, D. M.; PILGRIM, J. D.; KONSTANT, W. R.; FONSECA, G. A. B. Wilderness and biodiversity conservation. PNAS, Cambridge, v. 100, n. 18, p. 10309-10313, 2003.
MOURA, S. G.; PESSOA, F. B.; OLIVEIRA, F. F.; LUSTOSA, A. H. M.; SOARES, C. B. Animais silvestres recebidos pelo centro de triagem do IBAMA no Piauí no ano de 2011. Enciclopédia Biosfera, Goiânia, v. 8, n.15; p. 1748, 2012.

PAGANO, I. S. A.; SOUZA, A. E. B. A.; WAGNER, P. G. C.; RAMOS, R. T. C. Aves depositadas no Centro de Triagem de Animais Silvestres do IBAMA na Paraíba: uma amostra do tráfico de aves silvestres no estado. Ornithologia, Cabedelo, v. 3, n. 2, p. 133-144, 2009.

PIZO, M. A. Padrão de deposição de sementes e sobrevivência de sementes e plântulas de duas espécies de Myrtaceae na Mata Atlântica. Revista Brasileira de Botânica, São Paulo, v. 26, n. 3, p. 371-377, jul./set. 2003.

PORTAL BRASIL. Saiba mais sobre a fauna brasileira. 2012. Disponível em: <http://www. brasil.gov.br/meio-ambiente/2012/04/faunasilvestre>. Acesso em: 15 jun. 2015.

RENCTAS. Rede Nacional Contra o Tráfico Silvestre. $1^{\circ}$ Relatório nacional sobre o tráfico de fauna silvestre. Brasília, 2001.

SILVA, M. V.; SOUZA, M. B.; BERNARDE, P. S. Riqueza e dieta de serpentes do Estado do Acre, Brasil. Revista Brasileira de Zoociências, Juiz de Fora, v. 12, n. 2, p. 165-176, 2010. Disponível em: $<$ http://www.herpetofauna.com.br/Snakes Acre. pdf>. Acesso em: 3 nov. 2015.

SILVA, S. M.; LIMA, R. A. Levantamento da fauna silvestre no centro de reabilitação do batalhão da Polícia Militar Ambiental nos anos de 2010, 2011 e 2013 no Município de Candeias do JamariRO. Revista Eletrônica em Gestão, Educação e Tecnologia Digital, Santa Maria, v. 18, n. 1, p. 296311, abr. 2014. 
